\title{
Antimicrobial peptides secreted by equine mesenchymal stromal cells inhibit the growth of bacteria commonly found in skin wounds
}

Rebecca M. Harman, Steven Yang, Megan K. He and Gerlinde R. Van de Walle*

\begin{abstract}
Background: The prevalence of chronic skin wounds in humans is high, and treatment is often complicated by the presence of pathogenic bacteria. Therefore, safe and innovative treatments to reduce the bacterial load in cutaneous wounds are needed. Mesenchymal stromal cells (MSC) are known to provide paracrine signals that act on resident skin cells to promote wound healing, but their potential antibacterial activities are not well described. The present study was designed to examine the antibacterial properties of MSC from horses, as this animal model offers a readily translatable model for MSC therapies in humans. Specifically, we aimed to (i) evaluate the in vitro effects of equine MSC on the growth of representative gram-negative and gram-positive bacterial species commonly found in skin wounds and (ii) define the mechanisms by which MSC inhibit bacterial growth.
\end{abstract}

Methods: MSC were isolated from the peripheral blood of healthy horses. Gram-negative E. coli and gram-positive S. aureus were cultured in the presence of MSC and MSC conditioned medium (CM), containing all factors secreted by MSC. Bacterial growth was measured by plating bacteria and counting viable colonies or by reading the absorbance of bacterial cultures. Bacterial membrane damage was detected by incorporation of N-phenyl-1-naphthylamine (NPN). Antimicrobial peptide (AMP) gene and protein expression by equine MSC were determined by RT-PCR and Western blot analysis, respectively. Blocking of AMP activity of MSC CM was achieved using AMP-specific antibodies.

Results: We found that equine MSC and MSC CM inhibit the growth of E. coli and S. aureus, and that MSC CM depolarizes the cell membranes of these bacteria. In addition, we found that equine MSC CM contains AMPs, and blocking these AMPs with antibodies reduces the effects of MSC CM on bacteria.

Conclusions: Our results demonstrate that equine MSC inhibit bacterial growth and secrete factors that compromise the membrane integrity of bacteria commonly found in skin wounds. We also identified four specific AMPs produced by equine MSC. The secretion of AMPs may contribute to the value of MSC as a therapy for cutaneous wounds in both horses and humans.

Keywords: Equine mesenchymal stromal cells, Antimicrobial peptides

\footnotetext{
* Correspondence: grv23@cornell.edu

Baker Institute for Animal Health, College of Veterinary Medicine, Cornell University, Ithaca, NY 14850, USA
} International License (http://creativecommons.org/licenses/by/4.0/), which permits unrestricted use, distribution, and reproduction in any medium, provided you give appropriate credit to the original author(s) and the source, provide a link to the Creative Commons license, and indicate if changes were made. The Creative Commons Public Domain Dedication waiver (http://creativecommons.org/publicdomain/zero/1.0/) applies to the data made available in this article, unless otherwise stated. 


\section{Background}

Chronic cutaneous wounds are a rapidly growing health care burden in human medicine. In the United States, chronic leg and foot ulcers alone affect 2.4-4.5 million people [1, 2]. Chronic skin wounds are defined as wounds that do not improve after 4 weeks or do not heal within 8 weeks [3]. Chronic wounds are classified into different categories and are caused by a variety of insults. A common feature of chronic wounds, regardless of initial cause, is colonization by pathogenic bacteria, which leads to an inflammatory host response and delayed wound healing $[4,5]$. Chronic wounds typically contain a diversity of bacterial species that may interact to form matrices on wound surfaces called biofilms. Biofilms are particularly problematic as they show greater resistance to traditional antibiotics (Abx) compared to planktonic cells of the same species [6].

Antibiotic-resistant organisms are a challenge for the field of medicine in general, and for health care workers caring for patients with chronic cutaneous wounds in particular [7]. Alternatives to conventional Abx are currently being explored, with a focus on finding compounds that can kill bacteria directly rather than by disrupting metabolic activity and proliferation. Bacteria can survive insults to metabolic and proliferative pathways and evolve to avoid them, but they are less likely to become resistant to compounds that kill them directly $[7,8]$. Naturally occurring or synthetic antimicrobial peptides (AMPs) could be used for the design of new classes of Abx [9-11]. The diverse array of AMPs acts through different mechanism, and because many AMPs are bactericidal as opposed to bacteriostatic, it is unlikely that bacteria will be able to respond to these AMPs by adopting resistance strategies [12-14]. This makes AMPs a promising class of molecules to be explored as novel antimicrobial therapies. A disadvantage of synthetic AMPs as an alternative to conventional Abx, however, is that they are costly to generate and purify [15]. Therefore, naturally occurring AMPs for therapeutic use may be a more practical and cost-effective substitute for traditional antibiotic therapy.

To explore this, a suitable animal model that allows for proper testing of the efficacy of naturally occurring AMPs to kill bacteria in chronic wounds of humans is warranted. Like humans, horses suffer from naturally occurring chronic wounds $[16,17]$ and bacterial infection of horse wounds delays the normal healing process by prolonging inflammation, reducing resident skin cell migration, and disrupting extracellular matrix formation $[5,18]$.

Mesenchymal stromal cells (MSC) are adult multipotent progenitor cells, present in a variety of tissues and organs [19] and contribute to healing processes by participating in the inflammatory, proliferative and remodeling phases of tissue repair [20-22]. Recent data show that paracrine signaling is the primary mechanism by which MSC contribute to tissue repair [23, 24]. Our laboratory has previously demonstrated that equine MSC conditioned medium (CM), comprising all factors secreted by MSC, can increase equine dermal fibroblast migration and block the effects of transforming growth factor beta- 1 on equine dermal fibroblasts, providing the rationale for their potential in cutaneous wound management $[25,26]$. The potential of equine MSC to contribute to wound healing by reducing bacterial loads has not been explored to date, although this seems achievable based on previous reports showing that human MSC possess antimicrobial properties such as killing Escherichia coli (E. coli) in rodent lung infection models, as well as reducing overall bacterial loads in septic mice [27-29].

Therefore, the aims of the present study were to (i) evaluate the in vitro effects of equine MSC on the growth of representative gram-negative and gram-positive bacterial species commonly found in skin wounds and (ii) define the mechanisms by which MSC inhibit bacterial growth. Our notable findings were that equine MSC and MSC CM can inhibit the growth of E. coli and Staphylococcus aureus (S. aureus) and that MSC CM can depolarize the cell membranes of these bacteria. In addition, we identified four AMPs produced by MSC, and observed that blocking these AMPs in MSC CM with antibodies reduces the effects of MSC CM on bacteria.

\section{Methods \\ Cells}

Equine MSC were isolated from the peripheral blood of three healthy warmblood mares between 8 and 12 years old, exactly as described previously [30]. The blood collection was approved by the Cornell Institutional Animal Care and Use Committee (IACUC \# 2014-0038). Cells were seeded at a density of $1.6 \times 10^{5} \mathrm{cells} / \mathrm{cm}^{2}$ in a T75 flask in culture medium, consisting of low glucose Dulbecco's modified Eagle medium (DMEM) (Life Technologies, Grand Island, NY, USA) supplemented with $30 \%$ fetal bovine serum (FBS) (Atlanta Biological, Flowery Branch, GA, USA), $10^{-7} \mathrm{M}$ low dexamethasone (Sigma-Aldrich, St. Louis, MO, USA), $50 \mu \mathrm{g} / \mathrm{ml}$ gentamycin, $1 \times$ penicillin-streptomycin $(\mathrm{P} / \mathrm{S})$, and $2 \mathrm{mM} \mathrm{L}$ glutamine (all from Life Technologies). Cultures were maintained at $37{ }^{\circ} \mathrm{C}$ with $5 \% \mathrm{CO}_{2}$. At $70 \%$ confluency, cells were removed from flasks using $0.25 \%$ trypsinEDTA and further cultured in expansion medium, which is identical to the culture medium but without dexamethasone. Equine MSC were characterized by immunophenotypical protein profiling using flow cytometry and their potential for trilineage differentiation, exactly as described previously [31].

The equine dermal fibroblast line NBL6 (ATCC, Manassas, VA, USA) was cultured in standard medium, 
consisting of DMEM supplemented with 10\% FBS and $1 \times \mathrm{P} / \mathrm{S}$, and maintained at $37{ }^{\circ} \mathrm{C}$ with $5 \% \mathrm{CO}_{2}$.

\section{Bacterial cultures}

E. coli 10536 and S. aureus 25923 (ATCC) colonies were maintained on Luria-Bertani (LB) agar (Life Technologies) plates at $4{ }^{\circ} \mathrm{C}$ for up to 1 month. For each experiment, a colony of the appropriate species was picked and used to inoculate $4 \mathrm{ml} \mathrm{LB}$ broth (Life Technologies), which was incubated on a shaker at $200 \mathrm{rpm}$, overnight at $37^{\circ} \mathrm{C}$, in a warm room with ambient air. Overnight cultures were diluted 1:100 in $4 \mathrm{ml} \mathrm{LB}$ broth and allowed to incubate, shaking at $200 \mathrm{rpm}$, at $37{ }^{\circ} \mathrm{C}$ until cultures reached the exponential growth phase, as determined by the absorbance reading of $1 \mathrm{ml}$ culture at $600 \mathrm{~nm}$ using an Ultraspec 2100 pro spectrophotometer (Amersham Pharmacia Biotech, Cambridge, UK). Bacteria in the exponential growth phase were used for all experiments, unless stated otherwise.

\section{MSC-bacterial co-cultures}

For experiments in which MSC and bacteria were cocultured in direct contact with each other, 150,000 MSC or control NBL6 cells were plated per well in six-well plates in expansion or standard culture medium, respectively. After 24 hours (h), culture medium was removed, cell monolayers were rinsed twice with phosphatebuffered saline (PBS) and $1 \mathrm{ml}$ DMEM was added to wells. Bacteria were added at $1.5 \times 10^{6}$ per well. Control cultures contained bacteria in plain DMEM or DMEM with $2 \times \mathrm{P} /$ $\mathrm{S}$ without eukaryotic cells. All cultures were incubated for $6 \mathrm{~h}$ at $37^{\circ} \mathrm{C}$ in a warm room with ambient air, while shaking at $100 \mathrm{rpm}$. The $\mathrm{pH}$ of the culture medium was measured at the start and end of the incubation period, and remained constant at a $\mathrm{pH}$ of 7.5 throughout the experiments. Culture media and cell monolayers, lysed with $1 \%$ saponin (Sigma-Aldrich) in distilled water, from each well were transferred to $5 \mathrm{ml}$ tubes, vortexed to evenly distribute bacteria, and subsequently diluted in tenfold dilutions ranging from $1: 10$ to $1: 1,000,000$. Three $10 \mu \mathrm{l}$ drops of each dilution were spotted on LB agar plates and allowed to incubate overnight at $37{ }^{\circ} \mathrm{C}$. Bacterial colonies were counted and colony-forming units (CFU) per $\mathrm{ml}$ were calculated for each treatment.

Transwell experiments were carried out using the same numbers of cells and bacteria as were used for the direct contact co-cultures. For these assays, MSC or NBL6 cells were plated in $0.4 \mu \mathrm{m}$ transwell inserts (Corning, Oneonta, NY, USA) fitted in six-well culture plates. Bacteria were added to lower chamber and, after incubation for $6 \mathrm{~h}$ at $37^{\circ} \mathrm{C}$ while shaking at $100 \mathrm{rpm}$, in a warm room with ambient air, culture medium from the lower chamber was collected for assessment of live bacteria, as described above.

\section{Conditioned medium (CM) collection and treatments}

CM was collected from MSC and NBL6 cells after 2 days of culture, when cells were $70 \%$ confluent. To this end, $6 \times 10^{5}$ cells were seeded in a T75 flask with expansion medium. After $24 \mathrm{~h}$, medium was removed, cell monolayers were rinsed twice with $\mathrm{PBS}$, and $8 \mathrm{ml}$ DMEM were added. Medium was collected $24 \mathrm{~h}$ later, centrifuged twice for $7 \mathrm{~min}$ at $300 \times \mathrm{g}$ to remove cellular debris, and used for subsequent experiments.

Experiments were also performed with equine MSC $\mathrm{CM}$ that was treated as follows: to inactivate large secreted proteins, $\mathrm{CM}$ was heat inactivated at $80{ }^{\circ} \mathrm{C}$ for $30 \mathrm{~min}$ or treated with $1 \mathrm{U} / \mathrm{ml}$ proteinase $\mathrm{K}$ (Qiagen, Valencia, CA, USA) for $6 \mathrm{~h}$ at $37{ }^{\circ} \mathrm{C}$ before use. To determine if the active factors responsible for the antibacterial effects of MSC CM are biologically stable, CM was frozen and thawed, or lyophilized and reconstituted before being used in assays. To determine the active subfraction of the CM responsible for inhibiting bacterial growth, CM was filtered using Amicon Ultra-15 centrifugal filters (EMD Millipore, Darmstadt, Germany), as per manufacturer's instructions, and individual fractions containing secreted factors of specific molecular weights were used for subsequent experiments. To confirm the bioactive roles of identified AMP, CM was incubated with primary rabbit monoclonal antibodies against cystatin C (clone EPR4413) or rabbit polyclonal antibodies against elafin, lipocalin 2, cathelicidin (Abcam, Cambridge, MA, USA), combined equally for a final concentration of $4 \mu \mathrm{g} / \mathrm{ml}$, for $1 \mathrm{~h}$ prior to the start of the experiments. CM incubated with $4 \mu \mathrm{g} / \mathrm{ml}$ rabbit IgG (Abcam) was used as a control.

\section{CM-bacterial co-cultures}

CM collected as described above, was diluted 1:2 in LB broth for a total volume of $200 \mu \mathrm{l}$, and plated in triplicate wells of 96-well plates. Five hundred bacteria were added per well. Plates were incubated shaking at $100 \mathrm{rpm}$ at $37^{\circ} \mathrm{C}$, in a warm room with ambient air, for $8 \mathrm{~h}$ and $16 \mathrm{~h}$ for $E$. coli and $S$. aureus, respectively. Wells containing DMEM diluted 1:2 in LB (negative control) and DMEM diluted 1:2 in LB with $2 \times \mathrm{P} / \mathrm{S}$ (positive control) were also included. For the first experiment, the absorbance of the cultures was read using a 96-well Multiskan EX plate reader (Thermo Fisher Scientific, Waltham, MA, USA) at $600 \mathrm{~nm}$ and relative bacterial growth was calculated by comparing the absorbance of the CM wells or antibiotic-positive control wells to the absorbance of the DMEM-negative control wells. In addition, medium from each well was transferred to $1.5 \mathrm{ml}$ tubes, vortexed to evenly distribute bacteria, and subsequently diluted in tenfold dilutions, spotted on LB agar plates and allowed to incubate overnight at $37{ }^{\circ} \mathrm{C}$ as described above. Bacterial colonies 
were counted and CFU per $\mathrm{ml}$ were calculated for each treatment. By comparing the two read-outs (CFU and absorbance), we determined that absorbance adequately reflected the CFU results, and used it as the read-out for subsequent CM-bacterial co-culture assays.

A subset of $\mathrm{CM}$ co-culture experiments was done using bacterial cultures in the post-exponential, stationary, growth phase CM. To this end, stationary bacterial cultures were washed with PBS, and bacterial pellets containing $1 \times 10^{9} \mathrm{CFU}$ E. coli or $1 \times 10^{8} \mathrm{CFU}$ S. aureus were resuspended in either $12.5 \mathrm{mg} / \mathrm{ml}$ polymixin $\mathrm{B}$, $50 \mathrm{mg} / \mathrm{ml}$ nisin, or MSC CM. After $1 \mathrm{~h}$ at $4{ }^{\circ} \mathrm{C}$, bacteria were rinsed twice with PBS by centrifugation, diluted in tenfold dilutions in PBS, spotted on LB agar plates and allowed to incubate overnight at $37{ }^{\circ} \mathrm{C}$, as described above. Bacterial colonies were counted and CFU per ml were calculated for each condition.

\section{Biofilm assays}

Biofilm assays were carried out based on a method described by O'Toole [32]. Briefly, $50 \mu \mathrm{l}$ of equine MSC and NBL6 cell CM was pipetted into triplicate wells of u-bottom microtiter plates. DMEM and DMEM with $2 \times \mathrm{P} / \mathrm{S}$ were included as negative and positive controls, respectively. An equal volume of bacteria from cultures in the exponential growth phase was added to each well, and plates were incubated for $72 \mathrm{~h}$ to allow for biofilm formation. E. coli biofilms were grown at room temperature, $S$. aureus biofilms were grown at $37^{\circ} \mathrm{C}$, in a warm room with ambient air. At 24 and $48 \mathrm{~h}$, $50 \mu \mathrm{l} \mathrm{CM}$ or control medium was added to appropriate wells. After $72 \mathrm{~h}$, medium was removed from wells and wells were rinsed $2 \times$ with distilled water. $E$. coli biofilms were stained with $2.5 \%$ safranin and $S$. aureus biofilms were stained with $0.1 \%$ crystal violet for $10 \mathrm{~min}$, after which stains were removed by rinsing biofilms twice with distilled water and allowed to air dry. Dye retained in biofilms was then solubilized using 30\% acetic acid, and transferred to wells of 96-well flat bottom microtiter plates. Absorbance of solubilized dye was measured at $550 \mathrm{~nm}$ on an Infinite 200 pro plate reader (Tecan, Morrisville, NC, USA).

\section{Bacterial membrane depolarization assays}

In brief, CM was added to an equal volume of DMEM in the first column of wells in a 96-well microtiter plate and titered in 1:2 dilutions in DMEM. One-Nphenylaphthylamine (NPN) (Sigma-Aldrich) was added to each well for a final concentration of $10 \mu \mathrm{m}$, and $50 \mu \mathrm{l}$ of bacteria from cultures in the exponential growth phase were added. Plates were analyzed immediately in an Infinite 200 pro plate reader (Tecan) using an excitation of $355 \mathrm{~nm}$ and emission of $444 \mathrm{~nm}$.

\section{Antibody arrays}

A human proteome profiler antibody array, previously shown to cross-react with horse proteins [31, 33] was used, as per manufacturer's instructions (R\&D Systems, Minneapolis, MN, USA), to screen CM from equine MSC and equine dermal fibroblasts for the presence of AMPs. Positive signals were visualized using the ChemiDoc MP Imaging system (Bio-Rad, Hercules, CA, USA), normalized to the background, and data were quantified by measuring the sum of the intensities of the pixels within the spot boundary pixel area using image analysis software (Image Laboratory 4.1; Bio-Rad).

\section{Reverse transcription-polymerase chain reaction (RT-PCR)} RNA was extracted from cells using an RNeasy Mini Plus kit (Qiagen) and cDNA was synthesized using M-MLV Reverse Transcriptase (USB, Cleveland, OH, USA), per manufacturer's protocols. Primers were designed using Primer3 software, based on sequences found in the National Center of Biotechnology Information (NCBI) GenBank and, where possible, primer sets spanned an intronic region to prevent amplification of genomic DNA (Table 1). RT-PCR using Taq DNA Polymerase (Life Technologies) was performed to amplify the AMP genes cystatin C (CST3), elafin (PI3), lipocalin $2(L C N 2)$, cathelicidin $(C A M P)$ and beta defensin $2(D E F B 4 A)$. Beta-2-microglobulin $(\beta 2 M)$ was included as a reference gene. PCR products were run on a $1.5 \%$ agarose gel containing GelRed intercalating dye (Thermo Fisher Scientific) at $97 \mathrm{~V}$ for $1 \mathrm{~h}$ and gels were imaged on a BioRad ChemiDoc MP system (Bio-Rad). Band intensities were measured using Bio-Rad Image Lab software and the

Table 1 Primers used for RT-PCR

\begin{tabular}{llll}
\hline Gene product & Abbreviation & Forward primer $\left(5^{\prime}-3^{\prime}\right)$ & Reverse primer $\left(5^{\prime}-3^{\prime}\right)$ \\
\hline Cystatin C & CST3 & TTCCTGTCACCGTACAGC & GCACAATGTCCGTGGTGAA \\
Elafin & PI3 & GAGAAGGCTGAGTGCCAGAG & ACCAGCGAATCATCTCCAG \\
Lipocalin 2 & LCN2 & TCAAGGATGACCAGTTCCAG & CCTTCCTGAAGAACACGATG \\
Cathelicidin & CAMP & GGGTAGATGGTCACTGTTGC & AGCCCATTCTCCTTGAAGTC \\
Beta defensin 2 & CGTTCCTCGTTGTCTTCCT & CACAGGTGCCAATCTGTTTC \\
Beta-2-microglobulin & DEFB4A & GGGCTACTCTCCCTGACTGG & TACCTGCCCACACAGGTCAA \\
\hline
\end{tabular}


intensities of the AMP gene bands were divided by the intensity of reference gene bands, to calculate relative band intensities.

\section{Western blot and immunocytochemistry (ICC) analyses}

Western blot analyses were performed, exactly as previously described [26], using the same Ab that were used to block AMP activity in MSC CM, diluted 1:500, followed by horseradish peroxidase (HRP)-conjugated goat anti rabbit secondary Ab (Jackson ImmunoResearch Labs, West Grove, PA, USA), diluted 1:20,000.

ICC was performed, exactly as previously described [34], using the same Ab that were used to block AMP activity in MSC CM, diluted 1:100, followed by HRPconjugated goat anti rabbit secondary Ab, diluted 1:100.

\section{Statistical analyses}

The Student's $t$ test for unpaired data was used to test for statistically significant differences in relative bacterial growth (filtration experiments), and relative band intensities (RT-PCR and Western blot analyses). When multiple $t$ tests were performed on a single response variable within an experiment, the Holm-Šídák method was used to counteract the problems associated with multiple comparisons. One-way ANOVA, followed by the Tukey's multiple comparison test was used to determine statistically significant differences in bacterial viability (co-culture experiments, $\mathrm{CM}$ experiments and bactericidal versus bacteriostatic effects of MSC CM experiments), relative growth (biofilm, CM experiments excluding filtration experiments, antibody-blocking experiments and NPN experiments, excluding filtration experiments. GraphPad software was used for analysis (GraphPad Software, La Jolla, CA, USA). Data given are the mean of three replicates and the bars show standard deviations.

\section{Results}

Equine mesenchymal stromal cells (MSC) inhibit bacterial growth via paracrine signaling

To begin assessing the antimicrobial potential of equine MSC, we designed in vitro experiments to determine if MSC can inhibit the growth of representative gram-positive and gram-negative bacteria, E. coli and S. aureus, respectively, that are commonly found in cutaneous wounds. Plain medium (DMEM) and antibiotics (Abx) were included as negative and positive controls, respectively, and experiments were also performed with the equine dermal fibroblast cell line NBL6, since skin fibroblasts are known to secrete antimicrobial compounds [35-37].

When culturing bacteria in direct contact with equine MSC for 6 hours, like experiments previously carried out by Sung et al. with human MSC [38], we found that the growth of both bacterial species was significantly inhibited when compared to DMEM (Fig. 1a). Based on these encouraging results, we repeated these experiments with equine MSC cultured in transwell inserts to determine if the observed inhibition of bacterial growth was dependent on direct cell-to-cell contact. Equine MSC cultured in transwell inserts were still capable of effectively inhibiting the growth of bacteria within the time frame of the experiment (Fig. 1b), indicating that the observed effects are at least in part mediated by factors secreted by equine MSC, similar to what has been reported for human MSC [27, 38]. To determine whether equine MSC constitutively secrete factors with antimicrobial properties or if secretion is induced upon bacterial sensing, we cultured bacteria in $\mathrm{CM}$ collected from MSC and found that the relative bacterial growth, as assessed by CFU after $8 \mathrm{~h}$ for E. coli, and after $16 \mathrm{~h}$ for $S$. aureus, was significantly inhibited in the presence of MSC CM when compared to DMEM (Fig. 1c(i)). These results were directly compared to an alternative read-out for bacterial growth inhibition, namely the measurement of absorbance of bacterial cultures at $600 \mathrm{~nm}$ using a spectrophotometer. Although these two read-outs are not $100 \%$ similar (CFU/ml detects only live bacteria within a culture, while absorbance detects all bacteria that grew in the culture regardless of their viability at the time of measurement), the results obtained with absorbance reading reflected the results obtained by calculating $\mathrm{CFU} / \mathrm{ml}$ (Fig. 1c(ii)). Since absorbance measurements are less timeconsuming and allowed us to perform more technical replicates within each experiment, we decided to use absorbance as a read-out for subsequent $\mathrm{CM}$ experiments.

When comparing the results obtained with the equine MSC to the results obtained with the positive antibiotic (Abx) control and the NBL6 cells, we found that the levels of bacterial growth inhibition were not statistically different from the positive antibiotic control or NBL6 cells when we used $\mathrm{CFU} / \mathrm{ml}$ as a read-out, except that fewer $\mathrm{CFU} / \mathrm{ml} E$. coli were detected when cultured in MSC CM as compared to NBL6 CM (Fig. 1a-c). When using relative growth as a read-out, the results suggest that the levels of bacterial growth inhibition caused by equine MSC CM are situated between the levels of inhibition obtained by Abx and NBL6 cells (Fig. 1c). This may reflect the mechanism by which MSC-secreted factors inhibit bacterial growth.

Taken together, these results show that equine MSC secrete factors that effectively inhibit the growth of both $E$. coli and $S$. aureus, to levels comparable or even greater than those observed with Abx or dermal fibroblasts.

\section{Equine MSC secrete factors that inhibit bacterial biofilm formation}

Because biofilms contribute to the inhibition of cutaneous wound healing [7, 39] we assessed the effect of equine MSC CM on biofilm formation using an in vitro biofilm 


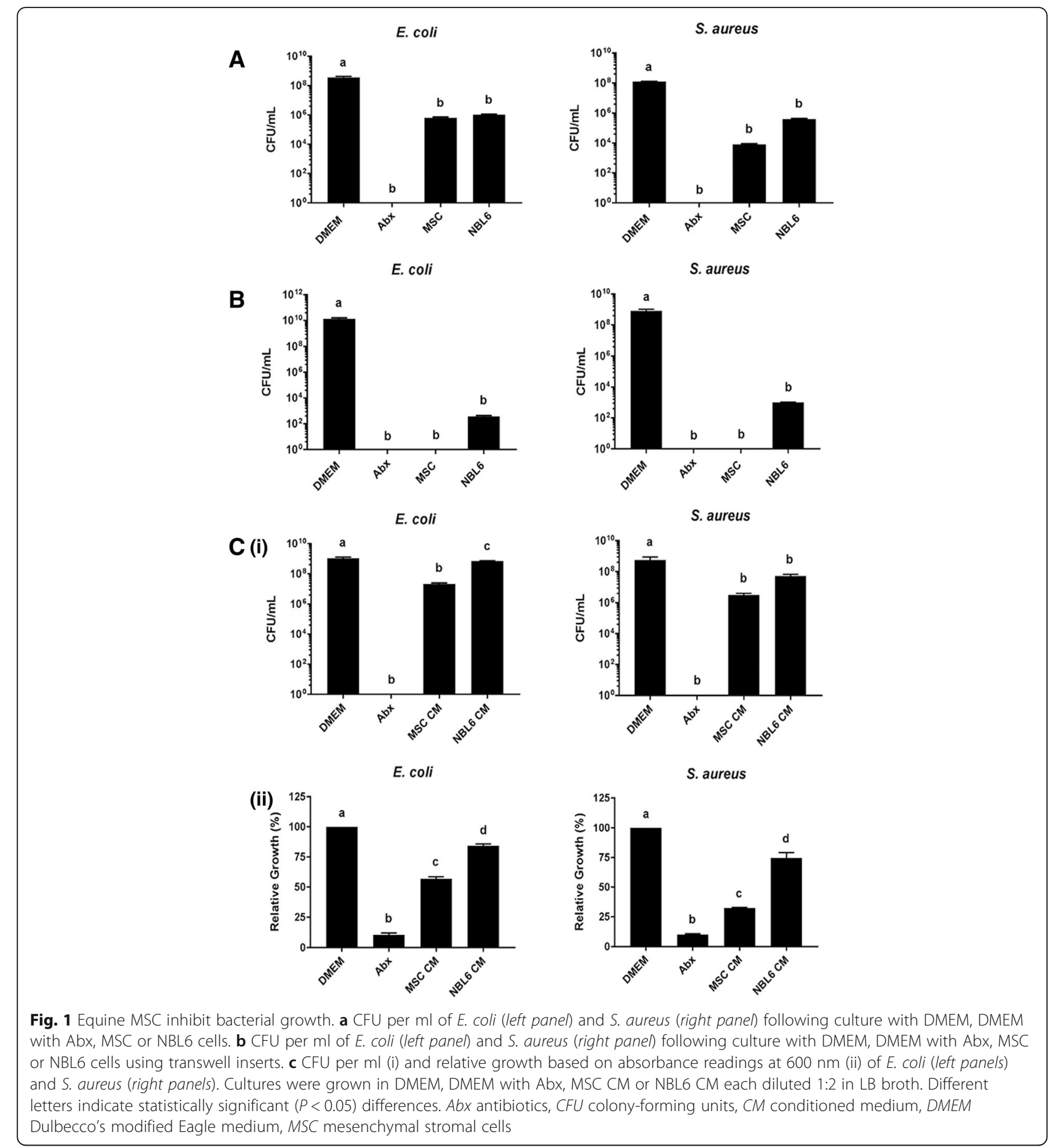

assay established by O'Toole [32]. Again, equine MSC CM significantly reduced the growth of both $E$. coli and $S$. aureus biofilms, to an extent that was virtually identical to that observed with the antibiotic control for both types of bacteria, and to levels that differed significantly from NBL6 CM for S. aureus (Fig. 2).
Equine MSC secrete stable, low molecular weight molecules that inhibit bacterial growth

To determine the characteristics of the bioactive factors with antimicrobial properties secreted by equine MSC, the following set of experiments was performed. First, we used boiling (heat inactivation $(\mathrm{HI})$ ) and proteinase $\mathrm{K}(\mathrm{PK})$ 
E. coli

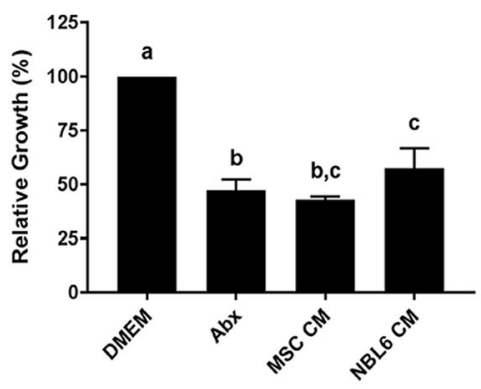

E. coli

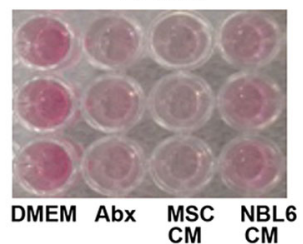

S. aureus

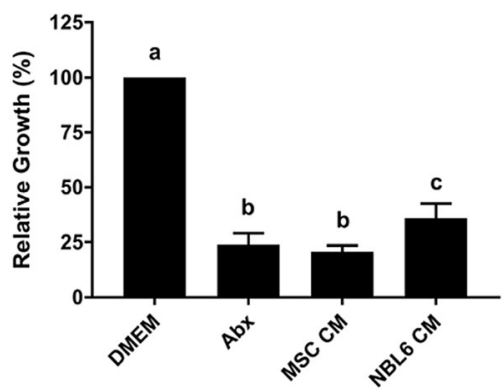

S. aureus

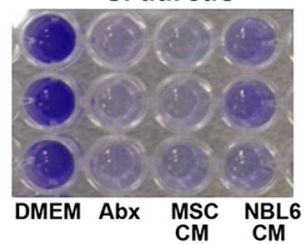

Fig. 2 Equine MSC constitutively secrete factors that inhibit bacterial biofilm formation. Relative growth of E. coli (left panel) and S. aureus (right panel) biofilms grown in DMEM, DMEM with Abx, MSC CM or NBL6 CM. Representative images of dye taken up by biofilms are shown below graphs. Different letters indicate statistically significant $(P<0.05)$ differences. $n=3$. Abx antibiotics, $C M$ conditioned medium, DMEM Dulbecco's modified Eagle medium, MSC mesenchymal stromal cells

treatment to inactive large proteins in the MSC CM, and compared the levels of bacterial growth inhibition to those obtained with untreated MSC CM. We found that HI CM still significantly inhibited the growth of both types of bacteria when compared to medium control, more specifically to levels indistinguishable from those obtained with the same MSC CM that was left untreated (Fig. 3a). Similar results were observed for the PK-treated CM, although the growth inhibition of $E$. coli was not as robust when compared to the untreated CM (Fig. 3a). Next, we froze and thawed, as well as lyophilized and reconstituted, CM before its use in the bacterial assays in order to determine the stability of the active factors. We observed that neither freezing nor lyophilizing significantly altered the growth inhibitory effects of CM on either bacterial species tested, as compared to fresh CM (Fig. 3b). Finally, we filtered the MSC CM to obtain subfractions containing secreted factors of specific sizes, and found that secreted factors of less than $10 \mathrm{kDa}$ and less than $30 \mathrm{kDa}$ significantly inhibited the growth of $E$. coli and $S$. aureus, respectively (dotted lines, Fig. 3c).

\section{Equine MSC secrete factors that depolarize bacterial cell} membranes, but do not appear to immediately kill bacteria In parallel, we decided to evaluate whether the observed MSC CM-mediated bacterial growth inhibition is caused by a membrane-depolarizing mechanism. To this end, we exposed bacteria to MSC CM in the presence of $1-\mathrm{N}$ phenylaphthylamine (NPN), a compound that is excluded by intact outer membranes of bacteria but taken up into the hydrophobic interior of outer membranes that have been depolarized. Consequently, a strong fluorescent NPN signal is correlated with disrupted bacterial membranes [40, 41]. The results from these experiments demonstrated that the equine MSC CM causes membrane depolarization of $E$. coli as well as $S$. aureus (i) in a concentration-dependent manner and (ii) to degrees similar to Abx known to depolarize gram-negative and grampositive bacterial membranes; polymixin $\mathrm{B}$ and nisin, respectively (Fig. 4a). Since polymixin B and nisin are bactericidal by killing bacteria shortly after contact [42, 43], we decided to evaluate whether the observed equine MSC CM-mediated bacterial membrane damage resulted in similar immediate killing of bacteria. To this end, we directly compared the number of viable bacteria after CM treatment to the numbers obtained after exposure to these two bactericidal Abx. Based on the difference in number of bacteria between these two types of treatments, with both bactericidal Abx resulting in significantly lower CFU/ $\mathrm{ml}(P<0.05$, Fig. $4 \mathrm{~b})$, we concluded that treatment of stationary, non-dividing bacteria with equine MSC CM did not immediately kill bacteria to the same extent as the bactericidal control compounds.

We then repeated the NPN experiments with the different CM subfractions and found that E. coli exposed to MSC-secreted factors greater than $10 \mathrm{kDa}$ took up more NPN than E. coli exposed to MSC-secreted factors smaller than $10 \mathrm{kDa}$, indicating that the bioactive factors that depolarize E. coli membranes are most likely $10 \mathrm{kDa}$ or greater in size (dotted line, Fig. 4c). Likewise, S. aureus 
E. coli

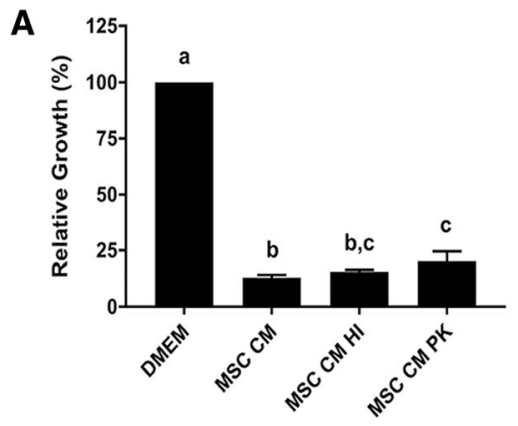

E. coli

B
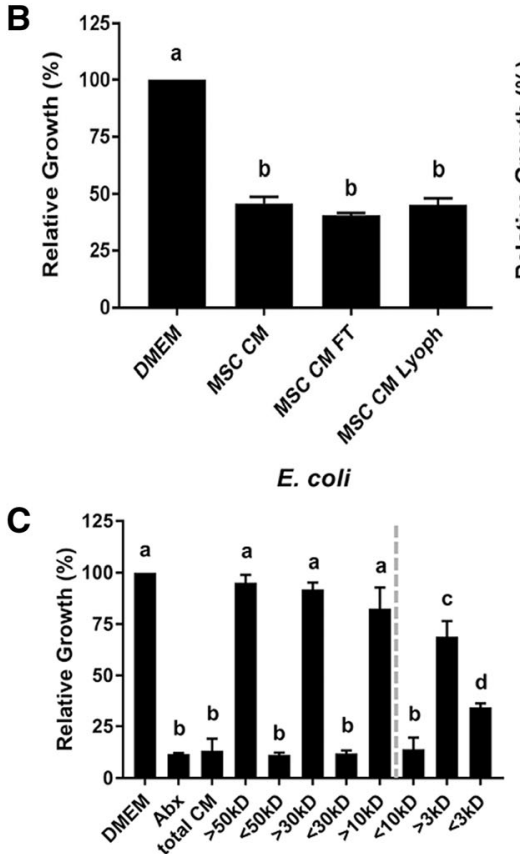

S. aureus

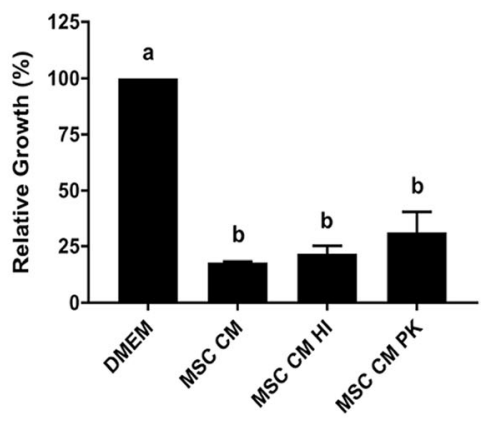

S. aureus

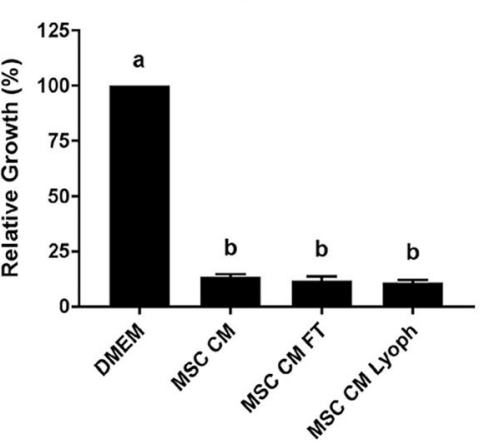

S. aureus

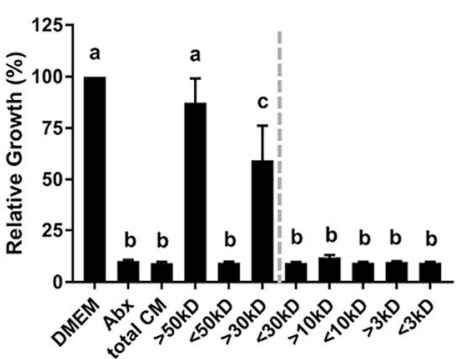

Fig. 3 Equine MSC secrete stable, low molecular weight factors that inhibit bacterial growth. a Relative growth of E. coli (left panel) and S. aureus (right panel) based on absorbance readings at $600 \mathrm{~nm}$. Cultures were grown in DMEM, MSC CM, heat-inactivated MSC CM (H) or proteinase K-treated CM (PK) each diluted 1:2 in LB broth. b Relative growth of E. coli (left panel) and S. aureus (right panel) based on absorbance readings at $600 \mathrm{~nm}$. Cultures were grown in DMEM, MSC CM, frozen-thawed MSC CM (FT) or lyophilized-reconstituted CM (Lyoph) each diluted 1:2 in LB broth. c Relative growth of E. coli (left panel) and S. aureus (right panel) based on absorbance readings at $600 \mathrm{~nm}$. Cultures were grown in DMEM, MSC CM, and MSC CM fractioned by size of secreted factors. Different letters indicate statistically significant $(P<0.05)$ differences. $\mathrm{n}=3$. Abx antibiotics, CM conditioned medium, DMEM Dulbecco's modified Eagle medium, MSC mesenchymal stromal cells

exposed to MSC-secreted factors greater than $30 \mathrm{kDa}$ took up more NPN than S. aureus exposed to MSCsecreted factors smaller than $30 \mathrm{kDa}$, indicating that the bioactive factors that depolarize $S$. aureus membranes are most likely $30 \mathrm{kDa}$ or greater in size (dotted line, Fig. 3c).

Collectively, these data indicate that equine MSC secrete a variety of bioactive factors of different sizes that affect bacteria by various modes of action, including membrane depolarization.

\section{Equine MSC secrete antimicrobial peptides (AMPs)}

Since AMPs are a class of low molecular weight molecules known to directly kill bacteria by forming pores in bacterial membranes $[13,14]$, we decided to evaluate the secretion of AMPs by equine MSC. We preliminarily screened the equine MSC CM, and a control dermal fibroblast CM for the presence of AMPs using a human proteome profiler array, which can detect the relative levels of over 100 proteins simultaneously $(n=1)$. The expression of three AMPs, namely cystatin $\mathrm{C}$, elafin, and lipocalin 2, were readily detected in MSC CM, at higher levels than in the control CM (Fig. 5a). The expression of these AMPs, as well as the expression of cathelicidin and beta defensin 2 (the most common mammalian AMPs, both of which have previously been described in the horse $[12,44])$, was then evaluated in the three 


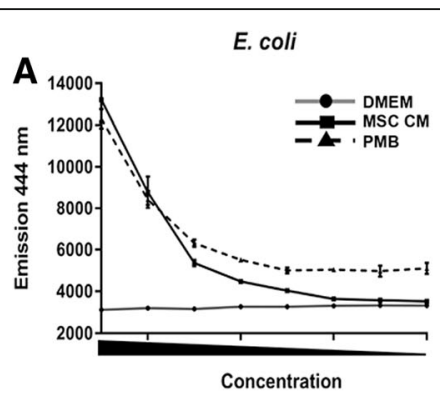

E. coli

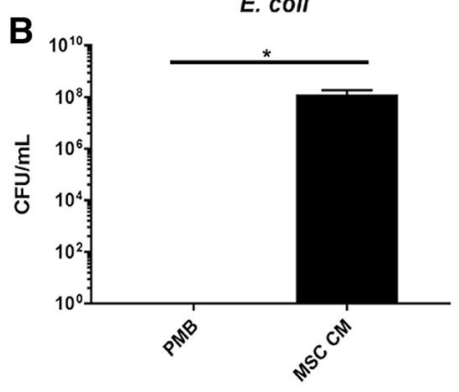

E. coli

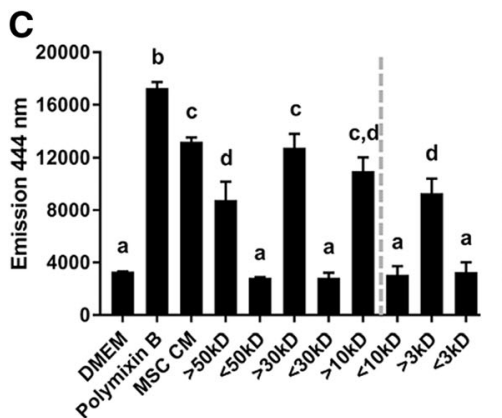

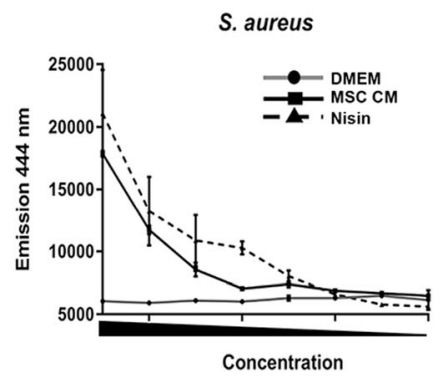

S. aureus

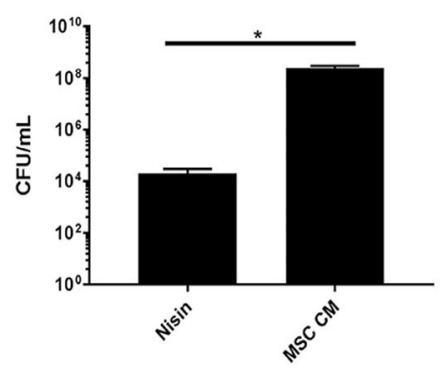

S. aureus

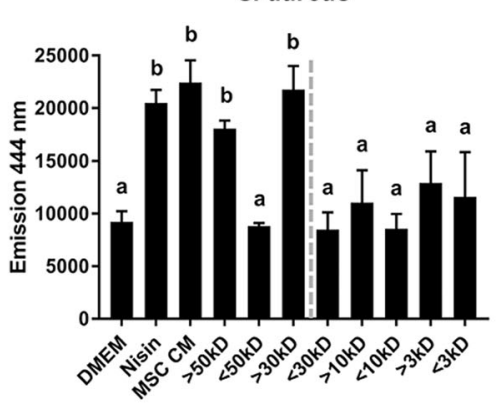

Fig. 4 Equine MSC secrete factors that depolarize bacterial cell membranes, but do not cause immediate cell death. a Incorporation of NPN into depolarized E. coli membranes (left panel) and S. aureus membranes (right panel) treated with MSC CM compared to high concentrations of compounds known to depolarize bacterial cell membranes. b CFU per ml of E. coli (left panel) and S. aureus (right panel) following culture at $4{ }^{\circ} \mathrm{C}$ with bactericidal compounds polymixin B (PMB), nisin or MSC CM. ${ }^{*} P<0.05$ c NPN incorporation by depolarized E. coli (left panel) and S. aureus (right panel) membranes as measured by fluorescent emission at $444 \mathrm{~nm}$. Bacteria were treated with DMEM, MSC CM, and MSC CM fractioned by size of secreted factors. Different letters indicate statistically significant $(P<0.05)$ differences. $n=3$. CFU colony-forming units, $C M$ conditioned medium, DMEM Dulbecco's modified Eagle medium, MSC mesenchymal stromal cells

equine MSC cultures that were used for the bacterial assays by RT-PCR using primers that had previously been confirmed by our laboratory to amplify products of the appropriate lengths in horse cells. Except for the gene $D E F B 4 A$ (encoding beta defensin 2), expression of the AMP transcripts CST3, PI1, LCN2, and CAMP (encoding cystatin $\mathrm{C}$, elafin, lipocalin and cathelicidin, respectively) could be detected (Fig. 5b). Next, Western blot analyses as well as immunocytochemistry (ICC) were performed to confirm protein expression of those AMPs that were detected on an mRNA level (Fig. 5c, d). For comparison, we also performed RT-PCR and Western blot analyses on NBL6 cells and found that whereas these cells showed similar expression levels of CST3, PI1, LCN2, and CAMP transcripts (Fig. 5b), the expression of the corresponding proteins cystatin $\mathrm{C}$, elafin, lipocalin and cathelicidin, was significantly lower in NBL6 compared to MSC cultures (Fig. 5c). This nicely corresponded with the original preliminary screening of dermal fibroblast $\mathrm{CM}$ using the human proteome profiler array (Fig. 5a).

The ability of equine MSC to inhibit bacterial growth and induce bacterial membrane damage is greatly reduced by pretreatment of CM with anti-AMP antibodies

To provide a link between the AMPs detected in equine MSC and the observed antimicrobial effects of the MSC $\mathrm{CM}$, we repeated the bacterial growth inhibition assays using MSC CM that was pretreated with antibodies against these AMPs. Although we found that anti-AMP antibody-pretreated $\mathrm{CM}$ still reduced bacterial growth 


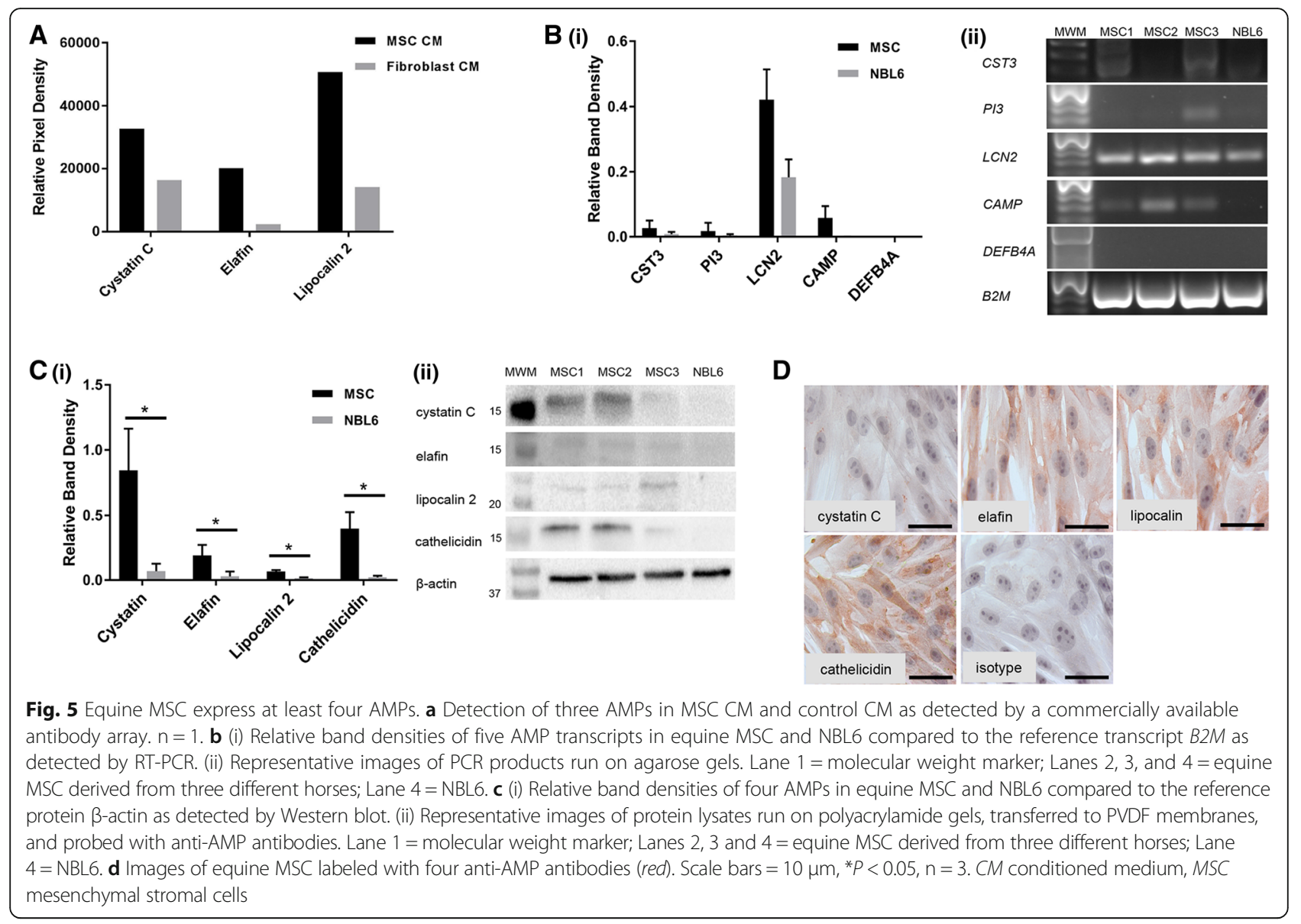

significantly when compared to the DMEM control (Fig. 6a), the bacterial growth inhibition by pretreated $\mathrm{CM}$ was significantly lower compared to untreated MSC CM or CM pretreated with isotype control antibodies (Fig. 6a). This suggests that cystatin C, elafin, lipocalin and/or cathelicidin are involved in, but not solely responsible for, the MSC CM-mediated growth inhibition of E. coli and S. aureus. We then corroborated these results by repeating the NPN experiments using antibody-pretreated MSC CM. A significant difference in E. coli membrane depolarization was observed compared to the DMEM control that was significantly less pronounced than the effects of untreated MSC CM or CM pretreated with isotype control antibodies (Fig. 6b (i and ii)). Interestingly, no significant difference in the membrane depolarization of $S$. aureus was observed between the antibody-pretreated MSC CM and untreated MSC $\mathrm{CM}$ or $\mathrm{CM}$ pretreated with isotype control antibodies, indicating that MSC-secreted factors other than cystatin C, elafin, lipocalin or cathelicidin are responsible for the membrane damage detected in this bacterial species (Fig. 6b (iii and iv)).

Taken together, these results demonstrate that the equine MSC secreted AMPs cystatin C, elafin, lipocalin and cathelicidin, are, at least in part, responsible for the observed growth inhibition of E. coli and S. aureus cultured in MSC CM. In addition, these AMPs also contribute to the membrane damage detected in E. coli, but not S. aureus.

\section{Discussion}

This study is the first to demonstrate that equine mesenchymal stromal cells (MSC) possess antibacterial properties by showing that MSC inhibit the growth of E. coli and $S$. aureus, and depolarize the membranes of these bacteria in vitro. Moreover, this study describes the presence of four distinct antimicrobial peptides (AMPs) in the equine MSC secretome, namely cystatin $\mathrm{C}$, elafin, lipocalin 2, and cathelicidin, and demonstrates that these AMPs are at least partially responsible for the antimicrobial effects of MSC.

The MSC-produced AMPs identified in this study represent several classes of antimicrobial compounds, some of which have been documented to be produced by MSC from other sources and species. Cystatin C has a mode of action that has not been fully elucidated to date, but has been identified as a secreted product of both murine bone marrow-derived and human adipose 

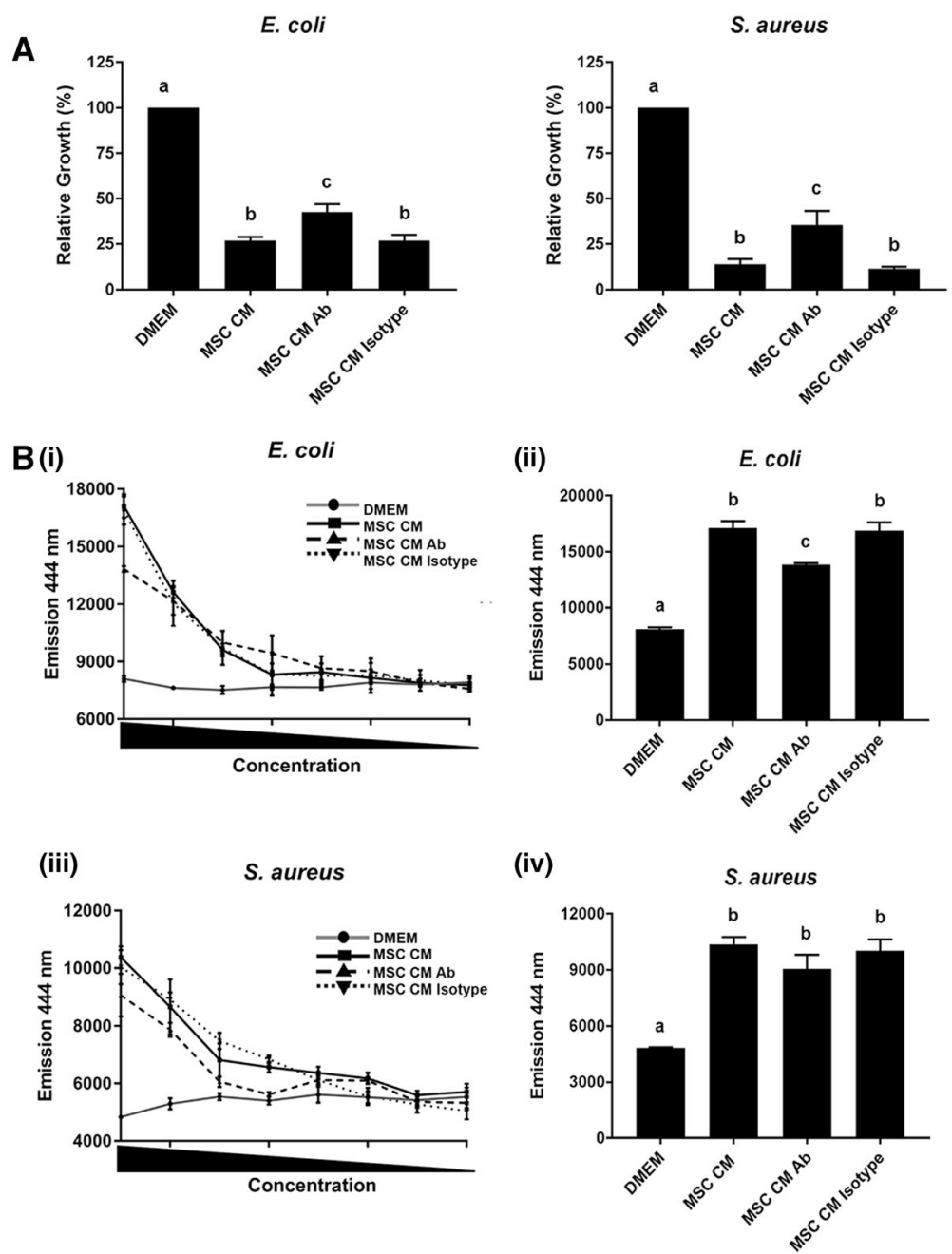

Fig. 6 Blocking AMP activity decreases the effects of MSC CM on bacteria. a Relative growth of E. coli (left panel) and S. aureus (right panel) based on absorbance readings at $600 \mathrm{~nm}$. Cultures were grown in DMEM, MSC CM, and MSC CM pre-incubated with anti-AMP antibodies (Ab) or MSC CM pre-incubated with an isotype control antibody, each diluted 1:2 in LB broth. b Incorporation of NPN into depolarized E. coli and S. aureus membranes in the presence of decreasing concentrations of DMEM, MSC CM, and MSC CM pre-incubated with anti-AMP antibodies or MSC CM pre-incubated with an isotype control antibody as measured by fluorescent emission at $444 \mathrm{~nm}$ (i and iii). Emission values from $E$. coli and $S$. aureus with treatments diluted 1:2 (ii and iv). Different letters indicate statistically significant $(P<0.05)$ differences. $n=3$. CFU colony-forming units, CM conditioned medium, DMEM Dulbecco's modified Eagle medium, MSC mesenchymal stromal cells

tissue-derived MSC [45, 46]. Lipocalin 2 limits bacterial growth by sequestering iron-containing siderophores and has been identified in the secretome of mouse bone marrow-derived MSC [47]. Cathelicidin is a cationic a that kills bacteria directly by disrupting membrane polarization and produces a cleavage product (LL-37) that has previously been described as a secreted product of human bone marrow-derived MSC [27, 48]. Elafin is known to kill bacteria by disrupting the membrane integrity. To our knowledge, elafin has not been reported as a product of MSC, so this study is the first to report on the production of this AMP by MSC.

Besides these four AMPs, we also evaluated the presence of beta defensin 2 in equine MSC since this AMP (i) has been described to be secreted by human umbilical cord-derived MSC [38, 49] and (ii) belongs to a ubiquitous family of AMPs that are found in most mammals, including horses [44, 50]. However, and to our surprise, we could not detect beta defensin 2 in equine MSC. This was not due to an improperly functioning of the PCR primers, as we have used the same primers to confirm expression of this AMP transcript in equine keratinocytes (data not shown). To identify the complete spectrum of equine MSC-derived AMPs, we plan to use a more unbiased and global approach (e.g., liquid chromatography-mass spectrometry (LC-MS/MS)) in future experiments.

The bacterial growth inhibition and biofilm assays were run in parallel with an equine dermal fibroblast cell 
line, based on the knowledge that the skin of most mammals produces AMPs $[50,51]$ and AMP mRNA and proteins have been identified in horse skin [44]. As expected, we found that equine dermal fibroblasts inhibit bacterial growth and produce specific AMPs. To the best of our knowledge, the AMP production by horse skin fibroblasts had not been evaluated previously. Interestingly, the expression of AMPs by the horse dermal fibroblast cell line NBL6 was not equivalent to that of equine MSC, with equine MSC producing higher levels of cystatin C, elafin, lipocalin 2 and cathelicidin. This further emphasizes the therapeutic potential of equine MSC in cutaneous wound management, as the administration of MSC or MSCsecreted products could supplement AMPs expressed at low levels in the skin, therefore expanding the range of AMPs locally present to fight skin infections.

Although the results of this study clearly demonstrate that equine MSC have antimicrobial properties against both $E$. coli and $S$. aureus, there appears to be a difference in the underlying mechanisms targeting each species. Based on the CM fractionation experiments, we demonstrated that secreted factors less than $10 \mathrm{kDa}$ in size are responsible for the observed growth inhibition of $E$. coli whereas secreted factors less than $30 \mathrm{kDa}$ appear to inhibit the growth of $S$. aureus. Moreover, MSC-secreted factors greater than $10 \mathrm{kDa}$ contributed to most membrane damage seen in E. coli, whereas secreted factors greater than $30 \mathrm{kDa}$ caused membrane damage to $S$. aureus. And although in the current study, we did not fully investigate the exact underlying mechanisms by which equine MSC CM affects different bacteria species, our results do clearly show that MSCsecreted factors (i) inhibit bacterial growth and (ii) depolarize the outer membranes of bacteria. It will be interesting, therefore, to study the effects of MSC CM on additional bacterial species commonly found in equine skin wounds, such as the gram-negative bacteria Pseudomonas aeruginosa and Acinetobacter baumannii, and the gram-positive Aerococcus viridians, Staphylococcus warneri, and Staphylococcus epidermidis [39], to determine whether there are indeed distinct mechanisms used by equine MSC to target gram-positive versus gram-negative bacteria. As most of these bacterial species are also found in human skin wounds [52, 53], results will be relevant to human as well as veterinary medicine.

Since we found that equine MSC secrete a variety of AMPs that appear effective against both gram-positive and gram-negative bacteria, these cells may serve as a broad-spectrum treatment to control bacterial growth and kill bacteria, without leading to resistance. Work by other groups has shown that in addition to controlling bacteria, AMPs and other host defense peptides directly contribute to wound healing by inducing cell migration and proliferation, promoting angiogenesis, and in general, accelerating the healing process [54]. This dual function of peptides to promote wound healing, combined with the ease and low cost of isolating MSC and collecting CM, makes MSC CM an ideal biological source for naturally occurring peptides as well as other factors that promote wound healing. Our data indicating that equine MSC CM can be lyophilized and still retain anti-bacterial activity suggest that using MSC CM therapeutically may be practical as well, providing a stable, off-the-shelf product for clinical use.

Taken together, our group focuses on the potential of equine MSC to be used as a therapy for skin wounds and the data generated in this study suggests that in addition to positively affecting resident skin cells, as we have demonstrated previously $[25,26,31]$, equine MSC may also improve cutaneous wound healing by reducing the bacterial load in wounds. Next, we intend to evaluate the efficacy of equine MSC in vivo by assessing how these cells promote skin wound healing and affect bacterial burden, in both acute and chronic wounds.

\section{Conclusions}

Mesenchymal stromal cells (MSC) have been reported to provide paracrine signals that promote cutaneous wound healing, but (i) the effects of equine MSC on the growth of gram-negative and gram-positive bacterial species commonly found in skin wounds and (ii) the mechanisms by which equine MSC inhibit bacterial growth had not been explored thus far. The present study is the first to show that equine MSC possess antimicrobial properties by inhibiting the growth of $E$. coli and $S$. aureus, in part by secreting antimicrobial peptides (AMPs) and depolarizing bacterial cell membranes. This antibacterial activity may contribute to the value of MSC as a therapy for chronic cutaneous wounds in both horses and humans, where colonization by pathogenic bacteria commonly inhibits normal healing.

\begin{abstract}
Abbreviations
Abx: Antibiotics; AMP: Antimicrobial peptide; CAMP: Cathelicidin; CFU: Colony-forming units; CM: Conditioned medium; CST3: Cystatin C; DEFB4A: Beta defensin 2; DMEM: Dulbecco's modified Eagle medium; FBS: Fetal bovine serum; HI: Heat inactivation; ICC: Immunocytochemistry; LB: Luria-Bertani; LCN2: Lipocalin 2; MSC: Mesenchymal stromal cells; NPN: 1N-phenylaphthylamine; P/S: Penicillin-streptomycin; PBS: Phosphate-buffered saline; PI3: Elafin; PK: Proteinase K; RT-PCR: Reverse transcription-polymerase chain reaction; $\beta 2 M$ : Beta-2-microglobulin
\end{abstract}

\section{Acknowledgements}

We gratefully acknowledge Drs. Helene Marquis and Cynthia Leifer for their technical advice.

\section{Funding}

This work was in part supported by an unrestricted fund to GVdW from the Harry M. Zweig Memorial Fund for Equine Research.

Availability of data and materials

The supporting data for this publication are available upon request. 


\section{Authors' contributions}

$\mathrm{RH}$ designed the study and is the main contributor of the article draft. RH, SY and $\mathrm{MH}$ carried out all laboratory procedures and analyzed the data. GVdW refined the study design, participated in data analyses, and revised the manuscript. All authors have read and approved the final manuscript.

\section{Competing interests}

The authors declare they have no competing interests.

\section{Consent for publication}

All authors provide consent for publication of this manuscript.

\section{Ethics approval and consent to participate}

Blood collection was approved in accordance with the Cornell Institutional Animal Care and Use Committee (IACUC \# 2014-0038).

\section{Publisher's Note}

Springer Nature remains neutral with regard to jurisdictional claims in published maps and institutional affiliations.

Received: 22 March 2017 Revised: 5 May 2017

Accepted: 14 June 2017 Published online: 04 July 2017

\section{References}

1. Brownrigg JRW, Apelqvist J, Bakker K, Schaper NC, Hinchliffe RJ. Evidencebased management of PAD \& the diabetic foot. Eur J Vasc Endovasc Surg. 2013:45:673-81.

2. Richmond NA, Maderal AD, Vivas AC. Evidence-based management of common chronic lower extremity ulcers. Dermatol Ther. 2013;26:187-96.

3. Mustoe TA, O'Shaughnessy K, Kloeters O. Chronic wound pathogenesis and current treatment strategies: a unifying hypothesis. Plast Reconstr Surg. 2006;117(7 Suppl):35S-41S.

4. Mustoe T. Understanding chronic wounds: a unifying hypothesis on their pathogenesis and implications for therapy. Am J Surg. 2004;187:565-70.

5. Rahim K, Saleha S, Zhu X, Huo L, Basit A, Franco OL. Bacterial contribution in chronicity of wounds. Microb Ecol. 2017;73:710-21. doi:10.1007/s00248-0160867-9.

6. Mah TF, OToole GA. Mechanisms of biofilm resistance to antimicrobial agents. Trends Microbiol. 2001;9:34-9.

7. Zhao G, Usui ML, Lippman SI, James GA, Stewart PS, Fleckman P, et al. Biofilms and inflammation in chronic wounds. Adv Wound Care. 2013;2:389-99.

8. Tillotson GS, Theriault N. New and alternative approaches to tackling antibiotic resistance. F1000Prime Rep. 2013:5:51. doi:10.12703/P5-51.

9. Marr AK, Gooderham WJ, Hancock RE. Antibacterial peptides for therapeutic use: obstacles and realistic outlook. Curr Opin Pharmacol. 2006;6:468-72.

10. Peters BM, Shirtliff ME, Jabra-Rizk MA. Antimicrobial peptides: primeval molecules or future drugs? PLoS Pathog. 2010;6:e1001067.

11. Lee $C-R$, Cho I, Jeong B, Lee $S$. Strategies to minimize antibiotic resistance. Int J Environ Res Public Health. 2013;10:4274-305.

12. Reddy KVR, Yedery RD, Aranha C. Antimicrobial peptides: premises and promises. Int J Antimicrob Agents. 2004;24:536-47.

13. Brogden KA. Antimicrobial peptides: pore formers or metabolic inhibitors in bacteria? Nat Rev Microbiol. 2005;3:238-50.

14. Bahar A, Ren D. Antimicrobial peptides. Pharmaceuticals. 2013;6:1543-75.

15. Reardon S. Bacterial arms race revs up. Nature. 2015;521:402-3.

16. Westgate SJ, Percival SL, Knottenbelt DC, Clegg PD, Cochrane CA. Chronic equine wounds: what is the role of infection and biofilms? Wounds. 2010; 22:138-45.

17. Theoret $\mathrm{CL}$, Wilmink JM. Aberrant wound healing in the horse: naturally occurring conditions reminiscent of those observed in man. Wound Repair Regen. 2013;21:365-71

18. Stashak TS, Theoret C, Stashak TS, editors. Equine wound management. 2nd ed. Ames: Wiley-Blackwell: Veterinary Wound Management Society/N. W.M.S; 2008.

19. Dittmer J, Leyh B. Paracrine effects of stem cells in wound healing and cancer progression (Review). Int J Oncol. 2014;44:1789-98.

20. Aggarwal S, Pittenger MF. Human mesenchymal stem cells modulate allogeneic immune cell responses. Blood. 2005;105:1815-22.

21. Gnecchi M, Zhang Z, Ni A, Dzau VJ. Paracrine mechanisms in adult stem cell signaling and therapy. Circ Res. 2008;103:1204-19.
22. Ono I, Yamashita T, Hida T, Jin H-Y, Ito Y, Hamada H, et al. Local administration of hepatocyte growth factor gene enhances the regeneration of dermis in acute incisional wounds. J Surg Res. 2004;120:47-55.

23. Baglio SR, Pegtel DM, Baldini N. Mesenchymal stem cell secreted vesicles provide novel opportunities in (stem) cell-free therapy. Front Physiol. 2012;3:359.

24. Madrigal M, Rao KS, Riordan NH. A review of therapeutic effects of mesenchymal stem cell secretions and induction of secretory modification by different culture methods. J Transl Med. 2014;12:240. doi:10.1186/s12967014-0260-8.

25. Bussche L, Harman RM, Syracuse BA, Plante EL, Lu Y-C, Curtis TM, et al. Microencapsulated equine mesenchymal stromal cells promote cutaneous wound healing in vitro. Stem Cell Res Ther. 2015;6:66. doi:10.1186/s13287015-0037-x.

26. Harman RM, Bihun IV, Van de Walle GR. Secreted factors from equine mesenchymal stromal cells diminish the effects of TGF- $\beta 1$ on equine dermal fibroblasts and alter the phenotype of dermal fibroblasts isolated from cutaneous fibroproliferative wounds. Wound Repair Regen. 2017;25:234-47.

27. Krasnodembskaya A, Song Y, Fang X, Gupta N, Serikov V, Lee J-W, et al. Antibacterial effect of human mesenchymal stem cells is mediated in part from secretion of the antimicrobial peptide LL-37. Stem Cells. 2010;28:2229-38.

28. Devaney J, Horie S, Masterson C, Elliman S, Barry F, O'Brien T, et al. Human mesenchymal stromal cells decrease the severity of acute lung injury induced by E. coli in the rat. Thorax. 2015;70:625-35.

29. Alcayaga-Miranda F, Cuenca J, Martin A, Contreras L, Figueroa FE, Khoury M. Combination therapy of menstrual derived mesenchymal stem cells and antibiotics ameliorates survival in sepsis. Stem Cell Res Ther. 2015;6:199. doi: 10.1186/s13287-015-0192-0.

30. Pennington MR, Curtis TM, Divers TJ, Wagner B, Ness SL, Tennant BC, et al. Equine mesenchymal stromal cells from different sources efficiently differentiate into hepatocyte-like cells. Tissue Eng Part C Methods. 2016;22: 596-607.

31. Bussche L, Van de Walle GR. Peripheral blood-derived mesenchymal stromal cells promote angiogenesis via paracrine stimulation of vascular endothelial growth factor secretion in the equine model. Stem Cells Transl Med. 2014:3:1514-25.

32. O'Toole GA. Microtiter dish biofilm formation assay. J Vis Exp. 2011. doi:10.3791/2437

33. Bara JJ, McCarthy HE, Humphrey E, et al. Bone marrow-derived mesenchymal stem cells become antiangiogenic when chondrogenically or osteogenically differentiated: Implications for bone and cartilage tissue engineering. Tissue Eng Part A. 2014;20:147-59.

34. Harman RM, Curtis TM, Argyle DJ, Coonrod SA, Van de Walle GR. A Comparative study on the in vitro effects of the DNA methyltransferase inhibitor 5-azacytidine (5-AzaC) in breast/mammary cancer of different mammalian species. J Mammary Gland Biol Neoplasia. 2016;21:51-66.

35. Liu L, Wang L, Jia HP, Zhao C, Heng HH, Schutte BC, et al. Structure and mapping of the human beta-defensin HBD-2 gene and its expression at sites of inflammation. Gene. 1998:222:237-44.

36. Schittek B, Hipfel R, Sauer B, Bauer J, Kalbacher H, Stevanovic S, et al. Dermcidin: a novel human antibiotic peptide secreted by sweat glands. Nat Immunol. 2001;2:1133-7.

37. Takahashi M, Horiuchi Y, Tezuka T. Presence of bactericidal/permeabilityincreasing protein in human and rat skin. Exp Dermatol. 2004:13:55-60.

38. Sung DK, Chang YS, Sung SI, Yoo HS, Ahn SY, Park WS. Antibacterial effect of mesenchymal stem cells against Escherichia coli is mediated by secretion of beta- defensin- 2 via toll- like receptor 4 signaling: Antibacterial effects of MSCs via beta defensin-2. Cell Microbiol. 2016;18:424-36.

39. Westgate SJ, Percival SL, Knottenbelt DC, Clegg PD, Cochrane CA. Microbiology of equine wounds and evidence of bacterial biofilms. Vet Microbiol. 2011:150:152-9.

40. Loh B, Grant C, Hancock RE. Use of the fluorescent probe 1-Nphenylnaphthylamine to study the interactions of aminoglycoside antibiotics with the outer membrane of Pseudomonas aeruginosa. Antimicrob Agents Chemother. 1984;26:546-51.

41. Bellemare A, Vernoux N, Morin S, Gagné SM, Bourbonnais Y. Structural and antimicrobial properties of human pre-elafin/trappin-2 and derived peptides against Pseudomonas aeruginosa. BMC Microbiol. 2010;10:253.

42. Henning S, Metz R, Hammes WP. Studies on the mode of action of nisin. Intl J Food Microbiol. 1986;3:121-34. 
43. Zavascki AP, Goldani LZ, Li J, Nation RL. Polymyxin B for the treatment of multidrug-resistant pathogens: a critical review. J Antimicrob Chemother. 2007;60:1206-15.

44. Bruhn O, Grötzinger J, Cascorbi I, Jung S. Antimicrobial peptides and proteins of the horse - insights into a well-armed organism. Vet Res. 2011;42:98.

45. Maurer MH. Proteomic definitions of mesenchymal stem cells. Stem Cells Int. 2011;2011:1-9.

46. Kalinina N, Kharlampieva D, Loguinova M, Butenko I, Pobeguts O, Efimenko A, et al. Characterization of secretomes provides evidence for adiposederived mesenchymal stromal cells subtypes. Stem Cell Res Ther. 2015;6:221.

47. Gupta N, Krasnodembskaya A, Kapetanaki M, Mouded M, Tan X, Serikov V, et al. Mesenchymal stem cells enhance survival and bacterial clearance in murine Escherichia coli pneumonia. Thorax. 2012;67:533-9.

48. Kościuczuk EM, Lisowski P, Jarczak J, Strzałkowska N, Jóźwik A, Horbańczuk J, et al. Cathelicidins: family of antimicrobial peptides. A review Mol Biol Rep. 2012;39:10957-70

49. Ganz T. Defensins: antimicrobial peptides of innate immunity. Nat Rev Immunol. 2003;3:710-20.

50. Hancock RE, Scott MG. The role of antimicrobial peptides in animal defenses. Proc Natl Acad Sci U S A. 2000;97:8856-61.

51. Schauber J, Gallo RL. Antimicrobial peptides and the skin immune defense system. J Allergy Clin Immunol. 2008;122:261-6.

52. Otto M. Staphylococcus colonization of the skin and antimicrobial peptides. Expert Rev Dermatol. 2010:5:183-95.

53. Gonzalez MR, Fleuchot B, Lauciello L, Jafari P, Applegate LA, Raffoul W, et al. Effect of Human Burn Wound Exudate on Pseudomonas aeruginosa Virulence. mSphere. 2016;1:e00111-5.

54. Niyonsaba F, Kiatsurayanon C, Chieosilapatham P, Ogawa H. Friends or Foes? Host defense (antimicrobial) peptides and proteins in human skin diseases. Exp Dermatol. 2017. doi:10.1111/exd.13314

\section{Submit your next manuscript to BioMed Central and we will help you at every step:}

- We accept pre-submission inquiries

- Our selector tool helps you to find the most relevant journal

- We provide round the clock customer support

- Convenient online submission

- Thorough peer review

- Inclusion in PubMed and all major indexing services

- Maximum visibility for your research

Submit your manuscript at www.biomedcentral.com/submit 